\title{
On Co-screen Conformality of 1-lightlike Submanifolds in a Lorentzian Manifold
}

\author{
Erol KILIÇ ${ }^{1}$, Sadık KELEŞ ${ }^{1} \&$ Mehmet GÜLBAHAR ${ }^{2}$ \\ ${ }^{1}$ Department of Mathematics, Faculty of Science and Art, İnönü University, Malatya, Turkey \\ 2 Department of Mathematics, Faculty of Science and Art, Siirt University, 56100, Siirt, Turkey \\ Correspondence: Mehmet GÜLBAHAR, Department of Mathematics, Faculty of Science and Art, 56100, Siirt \\ University, Turkey. Tel: 90-484-223-1224. E-mail: mehmetgulbahar85@gmail.com
}

Received: February 6, 2015 Accepted: March 2, 2015 Online Published: April 7, 2015

doi:10.5539/jmr.v7n2p76

URL: http://dx.doi.org/10.5539/jmr.v7n2p76

\begin{abstract}
In this paper, the co-screen conformal 1-lightlike submanifolds of a Lorentzian manifold are introduced as a generalization of co-screen locally half-lightlike submanifolds in (Wang, Wang \& Liu, 2013; Wang \& Liu, 2013) and two examples are given which one is co-screen locally conformal and the other is not. Some results are obtained on these submanifolds which the co-screen distribution is conformal Killing on the ambient manifold. The induced Ricci tensor of co-screen conformal 1-lightlike submanifolds is investigated.
\end{abstract}

Keywords: Lorentzian manifold, conformal killing, ricci tensor

Subject Clasification: 53C25, 53C50.

\section{Introduction}

Inspired by Einstein's theory of general relativity, the Kaluza-Klein's theory and the string theory, many physicists consider that the universe, we live in, can be a 4-dimensional submanifold embedded in high dimensional spacetime manifold and many mathematicians study not only submanifolds of Riemannian manifolds but also study semi-Riemannian manifolds. One can consider that semi-Riemannian submanifolds are two types which one is non-degenerate submanifolds and the other is lightlike submanifolds. In (Duggal \& Bejancu, 1996; Duggal \& Jin, 2007; Duggal \& Sahin, 2010), Duggal and his colleagues published books related with geometry of lightlike submanifolds and they presented general theory of lightlike submanifolds. Since then large numbers of papers have been published on lightlike submanifolds of semi-Riemannian manifolds.

Unfortunately, due to degenerate metric on lightlike submanifolds and the screen distribution is not canonical, induced nations of the submanifold (e.g sectional curvature, Ricci curvature, shape operator etc.) depend on choosing screen distribution that creates a problem. Therefore, it is necessary to find some classes of lightlike submanifold, whose geometry is essentially the same as that of their chosen screen distribution. Therefore, many mathematicians have been presented variety of methods to overcome this problem and have identified some special submanifolds. For example, the authors are used specific suitable methods for this problem in (Akivis \& Goldberg, 1998; Akivis \& Goldberg, 1999; Akivis \& Goldberg, 2000; Bolós, 2005; Bonnor, 1992; Leistner, 2006). Furthermore, Kupeli (Kupeli, 1996) has shown that any screen distribution of a lightlike submanifold is isometric to the factor bundle on the tangent space the submanifold. In (Atindogbe \& Duggal, 2004), Atindogbe and Duggal introduced screen locally conformal lightlike submanifold as a special lightlike submanifold of a semi-Riemannian manifold whose screen distribution is integrable and induced notions of the submanifold are independent of the screen distribution as follows:

A lightlike hypersurface $(M, g, S(T M))$ of a semi-Riemannian manifold is called screen locally conformal if there is the following relation between the shape operator $A_{N}$ and the local shape operator $A_{\xi}^{*}$ of the submanifold

$$
A_{N}=\varphi A_{\xi}^{*},
$$

where $\varphi$ is a non-vanishing smooth function on a neighborhood in $M$. 
Screen conformal lightlike submanifolds are studied on lightlike hypersurfaces in (Atindogbe, Ezin \& Tossa, 2006; Gülbahar, Kılıç \& Keleş, 2013a; Gülbahar, Kılıç \& Keleş, 2013b; Jin, 2009a; Jin, 2010; Jin, 2014) on half-lightlike submanifolds in (Duggal \& Sahin, 2004; Jin, 2009c) on coisotropic lightlike submanifolds in (Duggal, 2007), on indefinite complex space forms in (Jin, 2009b; Jin, 2010b), on indefinite contact space form in (Massamba, 2008; Massamba, 2012), on warped product manifold in (Sahin, 2005). Furthermore, screen conformal submersions are studied in (Sahin, 2007).

Recently, another special lightlike submanifolds whose screen distribution is integrable and the induced notions of the submanifold are independent of the screen distribution is defined in (Wang, Wang \& Liu, 2013; Wang \& Liu, 2013) and given by

$$
A_{u}=\varphi^{c} A_{\xi}^{*}
$$

where $\varphi^{c}$ is a non-vanishing smooth function on a neighborhood in $M, u$ is is a unit vector field of screen transversal bundle of the submanifold, $A_{u}, A_{\xi}^{*}$ are the shape operator and the local shape operator on $M$, respectively.

\section{Preliminaries}

Let $\widetilde{M}$ be a semi-Riemannian manifold equipped with semi-Riemannian metric $\widetilde{g}$ of index $\widetilde{q}$. The manifold $(\widetilde{M}, \widetilde{g})$ is called a Lorentzian manifold if $\widetilde{q}=1$.

Let $(M, g)$ be an $(n+1)$-dimensional lightlike submanifold of an $(n+m+2)$-dimensional Lorentzian manifold. The radical space $\operatorname{Rad} T_{p} M$ on the tangent space at a point $p \in M$ is one-dimensional subspace, defined by

$$
\operatorname{Rad} T_{p} M=\left\{\xi \in T_{p} M: g_{p}(\xi, X)=0, \forall X \in T_{p} M\right\}
$$

The submanifold $(M, g)$ is called a lightlike hypersurface if $m$ is equal to zero. The complementary vector bundle $S(T M)$ of $\operatorname{Rad} T M$ in $T M$ is called the screen bundle of $M$ (Duggal \& Bejancu, 2007). For these submanifolds, any screen bundle is non-degenerate and

$$
T M=\operatorname{Rad} T M \oplus_{\text {orth }} S(T M),
$$

where $\oplus_{\text {orth }}$ denotes the orthogonal direct sum. Consider a complementary vector bundle $S\left(T M^{\perp}\right)$ of $\operatorname{Rad} T M$ in $T M^{\perp}$. Then, we have the following orthogonal direct sum

$$
T M^{\perp}=\operatorname{Rad} T M \oplus_{\text {orth }} S\left(T M^{\perp}\right)
$$

Here, $S\left(T M^{\perp}\right)$ is the non-degenerate distribution with respect to $\widetilde{g}$. It is said to be co-screen distribution on $M$ (Jin, 2009c; Jin, 2010b).

Let $\operatorname{tr}(T M)$ and $\operatorname{lt}(T M)$ be complementary but not orthogonal vector bundles to $T M$ in $\left.T \widetilde{M}\right|_{M}$ and $\operatorname{Rad} T M$ in $\operatorname{tr}(T M)$, respectively. In this situation, we have

$$
\left.T \widetilde{M}\right|_{M}=(\operatorname{Rad} T M \oplus \operatorname{ltr}(T M)) \oplus_{\text {orth }} S(T M) \oplus_{\text {orth }} S\left(T M^{\perp}\right) .
$$

Let $(M, g)$ be an $(n+1)$-dimensional lightlike submanifold of an $(n+m+2)$ dimensional $(\widetilde{M}, \widetilde{g})$ and $\mathcal{U}$ be a local coordinate neighborhood of $M$. Then, there exists a quasi-orthonormal frame of $\widetilde{M}$ along $M$, on $\mathcal{U}$ :

$$
\left\{\xi, e_{1}, e_{2}, \ldots, e_{n}, N, u_{1}, u_{2}, \ldots, u_{m}\right\}
$$

where $\Gamma(S(T M))=\operatorname{Span}\left\{e_{1}, e_{2}, \ldots, e_{n}\right\}, \Gamma\left(\left.\operatorname{Rad} T M\right|_{\mathcal{U}}\right)=\operatorname{Span}\{\xi\}, \Gamma(\operatorname{ltr}(T M) \mid \mathcal{U})=\operatorname{Span}\{N\}$, $\Gamma(\operatorname{tr}(M) \mid \mathcal{u})=\operatorname{Span}\left\{u_{1}, u_{2}, \ldots, u_{m}\right\}$. We note that the quasi-orthonormal basis of $\widetilde{M}$ satisfies that

$$
\widetilde{g}(\xi, N)=1, \widetilde{g}(N, N)=\widetilde{g}(N, u)=\widetilde{g}(\xi, u)=0,
$$

for all $u \in \Gamma(\operatorname{tr}(T M) \mid \mathcal{u})$.

Let $\widetilde{\nabla}$ be the Levi-Civita connection of $\widetilde{M}$ and $P$ be the projection morphism of $\Gamma(T M)$ to $\Gamma(S(T M))$. The Gauss 
and Weingarten formulas are given by

$$
\begin{aligned}
& \widetilde{\nabla}_{X} Y=\nabla_{X} Y+B(X, Y) N+\sum_{\alpha=1}^{m} D^{\alpha}(X, Y) u_{\alpha}, \\
& \widetilde{\nabla}_{X} N=-A_{N} X+\rho N+\sum_{\alpha=1}^{m} \rho_{\alpha} u_{\alpha}, \\
& \widetilde{\nabla}_{X} u_{\beta}=-A_{u_{\beta}} X+\varepsilon N+\sum_{\alpha=1}^{m} \varepsilon_{\alpha} u_{\alpha}, \\
& \nabla_{X} P Y=\nabla_{X}^{*} P Y+C(X, P Y) \xi \\
& \nabla_{X} \xi=-A_{\xi} X-\rho \xi
\end{aligned}
$$

for any $X, Y \in \Gamma(T M)$, where $\nabla$ and $\nabla^{*}$ are the induced linear connections on $T M$ and $S(T M)$, respectively; $B$ and $D^{\alpha}$ are coefficients of the lightlike second fundamental form and coefficients of the screen second fundamental form of $T M$, respectively, $C$ is coefficients of the local second fundamental form on $S(T M), A_{N}, A_{u_{\beta}}$ are the shape operators on $M, A_{\xi}^{*}$ is the shape operator on $S(T M)$ and $\varepsilon, \varepsilon_{\alpha}, \rho, \rho_{\alpha}$ are 1 -forms on $M$.

Let us define a local 1-form $\eta$ by

$$
\eta(X)=\widetilde{g}(X, N), \forall X \in \Gamma\left(\left.T M\right|_{\mathcal{U}}\right)
$$

Consider (9), (14) and $\widetilde{\nabla}$ is a metric connection on $\widetilde{M}$ it is known that the induced connection $\nabla$ is not a metric connection (Duggal \& Bejancu, 2007).

The second fundamental form $h$ and the local second fundamental form $h^{*}$ are given by, respectively,

$$
h(X, Y)=h^{\ell}(X, Y)+h^{s}(X, Y) \text { and } h^{*}(X, P Y)=C(X, P Y) \xi
$$

where

$$
h^{\ell}(X, Y)=B(X, Y) N \text { and } h^{s}(X, Y)=\sum_{\alpha=1}^{m} D^{\alpha}(X, Y) u_{\alpha}
$$

for any $X, Y \in T M$. Here, we note that $h^{\ell}$ is the light part of the second fundamental form and $h^{s}$ is the nondegenerate part of the second fundamental form.

It is known that $B=0$ on $\operatorname{Rad} T M$ and it is independent of the screen distribution $S(T M)$ for any $\alpha \in\{1, \ldots m\}$ and the following relations satisfy that

$$
\begin{aligned}
& B(X, P Y)=g\left(A_{\xi}^{*} X, Y\right), \widetilde{g}\left(A_{\xi}^{*} X, N\right)=0, \\
& D^{\alpha}(X, P Y)=g\left(A_{u_{\alpha}} X, P Y\right), \widetilde{g}\left(\nabla_{X} N, u_{\alpha}\right)=\rho_{\alpha}, \\
& C(X, P Y)=g\left(A_{N} X, P Y\right),
\end{aligned}
$$

for any $X, Y \in T M$ and $\alpha \in\{1, \ldots, m\}$.

The submanifold $(M, g, S(T M))$ is called totally umbilical if there exist smooth functions $\lambda \in F(\operatorname{tr}(T M))$ and $\lambda_{\alpha} \in F\left(S(T M)^{\perp}\right)$ for any $\alpha \in\{1, \ldots, m\}$ such that

$$
B(X, Y)=\lambda g(X, Y) \text { and } D^{\alpha}(X, Y)=\lambda_{\alpha} g(X, Y)
$$

for all $X, Y \in \Gamma(T M)$.

A 1-lightlike submanifold is said to be irrotational if $\widetilde{\nabla}_{X} \xi \in \Gamma(T M)$ for any $X \in \Gamma(T M)$, where $\xi \in \Gamma(\operatorname{Rad} T M)$ (Kupeli, 1996).

Let $\left\{e_{1}, \ldots, e_{n}\right\}$ be an orthonormal basis of $\Gamma(S(T M))$ and define

$$
\mu^{*}=\frac{1}{n} \sum_{i=1}^{n} C\left(e_{i}, e_{i}\right), \quad \mu_{1}=\frac{1}{n} \sum_{i=1}^{n} B\left(e_{i}, e_{i}\right), \quad \mu_{2}=\frac{1}{n} \sum_{i=1}^{n} \sum_{\alpha=1}^{m} D^{\alpha}\left(e_{i}, e_{i}\right) .
$$


The manifold $(M, g, S(T M))$ is called minimal if $\mu_{1}=\mu_{2}=0$ and $D^{\alpha}=0$ on Rad TM (Bejan \& Duggal, 2005; Duggal \& Sahin, 2010).

The mean curvature vector on $T M$ and on $\Gamma(S(T M))$ are given by

$$
\begin{array}{r}
H(p)=\frac{1}{n} \operatorname{trace}_{S(T M)} h=\mu_{1} N+\sum_{\alpha=1}^{m} \mu_{2} u_{\alpha}, \\
H^{*}(p)=\mu^{*} \xi+\mu_{1} N+\sum_{\alpha=1}^{m} \mu_{2} u_{\alpha},
\end{array}
$$

respectively.

Let $\Pi=s p\left\{e_{i}, e_{j}\right\}$ be 2-dimensional non-degenerate plane of the tangent space $T_{p} M$ at $p \in M$. Then, the number

$$
K_{i j}=\frac{g\left(R_{p}\left(e_{j}, e_{i}\right) e_{i}, e_{j}\right)}{g_{p}\left(e_{i}, e_{i}\right) g_{p}\left(e_{j}, e_{j}\right)-g_{p}\left(e_{i}, e_{j}\right)^{2}}
$$

is called the sectional curvature of the section $\Pi$ at $p \in M$. Since the operator $C$ isn't symmetric the sectional curvature function doesn't need to be symmetric on any lightlike submanifold of a semi-Riemannian manifold (Duggal \& Sahin, 2010).

Let $\xi$ be a null vector of $T_{p} M$. A plane $\Pi$ of $T_{p} M$ is called a null plane if it contains $\xi$ and $e_{i}$ such that $\widetilde{g}\left(\xi, e_{i}\right)=0$ and $\widetilde{g}\left(e_{i}, e_{i}\right) \neq 0$. The null sectional curvature of $\Pi$ be given in (Beem, Ehrlich, \& Easley, 1996) as follows:

$$
K_{\xi}(\Pi)=\frac{g\left(R_{p}\left(e_{i}, \xi\right) \xi, e_{i}\right)}{g_{p}\left(e_{i}, e_{i}\right)} .
$$

We note that the null sectional curvature measures differences in length of two spacelike geodesic constructed from the degenerate plane section $\Pi$ and it is independent of the choice of the spacelike vector $e_{i}$ but it depends quadratically on the null vector $\xi$ (Albujer \& Haesen, 2010).

Let $(M, g, S(T M))$ be an $(n+1)$ dimensional 1-lightlike submanifold of an $\widetilde{m}$-dimensional Lorentzian manifold $(\widetilde{M}, \widetilde{g})$ and $\left\{e_{1}, \ldots \ldots, e_{n}\right\}$ be an orthonormal basis of $\Gamma(S(T M))$. The induced Ricci type tensor $R^{(0,2)}$ of $M$ is defined by

$$
R^{(0,2)}(X, Y)=\sum_{i=1}^{n} g\left(R\left(e_{i}, X\right) Y, e_{i}\right)+\widetilde{g}(R(\xi, X) Y, N) .
$$

It is known that $R^{(0,2)}$ is not symmetric and has no geometric meaning. The tensor $R^{(0,2)}$ is called the Ricci curvature if it is symmetric (Duggal \& Sahin, 2010).

\section{Co-screen Conformal 1-lightlike Submanifolds}

We begin this section with the canonical theorems for 1-lightlike submanifolds of a Lorentzian manifold.

Let $(M, g)$ be an $(n+1)$-dimensional 1-lightlike submanifold of an $(m+n+2)$-dimensional Lorentzian manifold $(\widetilde{M}, \widetilde{g})$. Let us consider two quasi-orthonormal frame $\left\{\xi, N, e_{i}, u_{\alpha}\right\}$ and $\left\{\xi, N^{\prime}, e_{i}^{\prime}, u_{\alpha}^{\prime}\right\}$ induced on $\mathcal{U}$. In this case, the followings can be written

$$
e_{a}^{\prime}=\sum_{b=1}^{n} e_{a}^{b}\left(e_{b}-f_{b} \xi\right), \quad u_{\alpha}^{\prime}=\sum_{\beta=1}^{m} u_{\alpha}^{\beta}\left(u_{\beta}-Q_{\beta} \xi\right)
$$

and

$$
N^{\prime}=N+N_{1} \xi+\sum_{a=1}^{n} f_{a} e_{a}+\sum_{\alpha=1}^{m} Q_{\alpha} u_{\alpha},
$$

where $e_{a}^{b}, u_{\alpha}^{\beta}, N_{1}, f_{a}$ and $Q_{\alpha}$ are differentiable functions on $\mathcal{U}$ for $a \in\{1, \ldots, n\}$ and $\alpha \in\{1, \ldots, m\}$. Since $\widetilde{g}(N, N)=0$, we have

$$
N_{1}=-\frac{1}{2} \sum_{a=1}^{n}\left(f_{a}\right)^{2}-\frac{1}{2} \sum_{\alpha=1}^{m}\left(Q_{\alpha}\right)^{2} .
$$


Let $h^{\ell}$ and $h^{\prime \ell}$ are light parts of the second fundamental forms and $B$ and $B^{\prime}$ are coefficients of the light parts of the second fundamental forms on screen distributions $S(T M)$ and $S(T M)^{\prime}$, respectively. Taking $\widetilde{\xi}=\theta \xi$ and thus $\widetilde{N}=\frac{1}{\theta} N$, where $\theta$ is some function, we obtain

$$
h^{\prime \ell}(X, Y)=\widetilde{g}\left(\nabla_{X} Y, \widetilde{\xi}\right) \widetilde{N}=\widetilde{g}\left(\nabla_{X} Y, \xi\right) N=h^{\ell}(X, Y),
$$

which implies that $h^{\ell}$ is independent of the screen distribution $S(T M)$.

Let $h^{s}$ and $h^{\prime s}$ are non-degenerate parts of the second fundamental forms and $D$ and $D^{\prime}$ are coefficients of the non-degenerate parts of the second fundamental forms on screen distributions $S(T M)$ and $S(T M)^{\prime}$, respectively. Using (26), we have

$$
\begin{aligned}
D^{\prime \alpha}(X, Y) & =\widetilde{g}\left(\widetilde{\nabla}_{X} Y, u_{\alpha}\right) \\
& =\sum_{\beta=1}^{m} u_{\alpha}^{\beta}\left(D^{\beta}(X, Y)-Q_{\beta} B(X, Y)\right),
\end{aligned}
$$

which implies that non-degenerate part of the second fundamental form $h^{s}$ depends on the screen distribution.

Using similar method, one can easily get

$$
C^{\prime}(X, P Y)=C(X, P Y)-\frac{1}{2}\|e\|^{2} B(X, Y)+g\left(\nabla_{X} P Y, e\right)
$$

where $e=\sum_{a=1}^{n} f_{a} e_{a}$ is called the characteristic vector field.

Let us consider the first derivative of a screen distribution $S(T M)$ given by

$$
S(p)=\operatorname{Span}\left\{[X, Y]_{p}: X_{p}, Y_{p} \in S(T M), p \in M\right\},
$$

where [, ] denotes the Lie-bracket. Then, we have the following:

Theorem 1 Let $(M, g, S(T M))$ be an $(n+1)$-dimensional 1-lightlike submanifold of a Lorentzian manifold. If the first derivative $S$ defined by (32) coincides with $S(T M)$, then $S(T M)$ is a canonical screen of $M$, up to an orthogonal transformation with a canonical lightlike transversal vector bundle and the screen second fundamental form $h^{*}$ is independent of a screen distribution.

The proof is same as that of Theorem 2.1 in (Duggal, 2007), so we omit it here.

Now, we recall a class of 1-lightlike submanifolds of a Lorentzian manifold which admits an integrable canonical screen distribution as follows.

Definition 2 (Atindogbe \& Duggal, 2004) A 1-lightlike submanifold $(M, g, S(T M))$ of a Lorentzian $(\widetilde{M}, \widetilde{g})$ is called a screen locally conformal if

$$
B(X, Y)=\varphi C(X, Y), \quad \forall X, Y \in \Gamma\left(\left.T M\right|_{\mathcal{U}}\right),
$$

where $\mathcal{U}$ is a local coordinate neighborhood of $M$ and $\varphi$ is a smooth function on a neighborhood $\mathcal{U}$ in $M$. If $\varphi$ is non-zero constant then the submanifold is called screen homothetic.

Now, we state the following theorem:

Theorem 3 Let $(M, g, S(T M))$ be an $(n+1)$-dimensional 1-lightlike submanifold of an $(n+m+2)$-dimensional Lorentzian manifold $(\widetilde{M}, \widetilde{g})$. Suppose that $S(T M)$ is integrable and $S(T M)$ is totally umbilical immersed in $\widetilde{M}$ and it is parallel along integral curves of the radical distribution. Then, $M$ is screen locally conformal if and only if $\mu^{*} \mu_{1} \neq 0$.

Proof. Let us denote $M^{\prime}$ as a leaf of $S(T M)$. Then, we have

$$
\nabla_{X} Y=\nabla_{X}^{*} Y+C(X, Y) \xi+B(X, Y) N+\sum_{\alpha=1}^{m} D^{\alpha}(X, Y) u_{\alpha}
$$

for all $X, Y \in T M^{\prime}$. The mean curvature vector of $M^{\prime}$ is a vector field of the rank $(m+2)$. From (22) and since $M^{\prime}$ is totally umbilical, it is clear that

$$
C(X, Y) \xi+B(X, Y) N+\sum_{\alpha=1}^{m} D^{\alpha}(X, Y) u_{\alpha}=g(X, Y)\left(\mu^{*} \xi+\mu_{1} N+\sum_{\alpha=1}^{m} \mu_{2} u_{\alpha}\right) .
$$


In other words,

$$
B(X, Y)=\mu_{1} g(X, Y), C(X, Y)=\mu^{*} g(X, Y) \text { and } D^{\alpha}(X, Y)=\mu_{2} g(X, Y),
$$

for any $\alpha \in\{1, \ldots, m\}$. Also, it is known that the mean curvature vector $H^{*}$ on $\Gamma(S(T M))$ satisfies that

$$
\widetilde{g}\left(H^{*}, H^{*}\right)=2 \mu_{1} \mu^{*}+\mu_{2}^{2} \geq 0 .
$$

If $\mu_{1} \mu^{*} \neq 0$ then $A_{N} X=\frac{\mu_{1}}{\mu^{*}} A_{\xi}^{*} X$ for all $X \in T M^{\prime}$. Since $S(T M)$ is parallel along integral curves of the radical distribution, we have $A_{N} \xi=0$ so that

$$
A_{N} X=\frac{\mu_{1}}{\mu^{*}} A_{\xi}^{*} X
$$

for all $X \in T M$ which implies that $M$ is screen locally conformal. The proof of the converse part is straightforward. Using same proof way of Theorem 2.3 in (Duggal \& Bejancu, 1996), we immediately have the following theorem:

Theorem 4 Let $(M, g, S(T M))$ be a 1-lightlike submanifold of a Lorentzian manifold. Then, the following assertions are equivalent:

1) $S(T M)$ is integrable ;

2) $h^{*}(X, Y)=h^{*}(Y, X)$ for all $X, Y \in \Gamma(S(T M))$;

3) the shape operator $A_{N}$ on $M$ is symmetric.

From the above theorem, it is clear that screen conformal 1-lightlike submanifolds have the important features that their screen distributions are always integrable and the sectional curvature function is always symmetric and it has significant geometric meanings as in Riemannian manifolds.

We give now the following definition that shows that there is another type lightlike submanifold which its screen distributions are always integrable and the sectional curvature function defined on it is always symmetric.

Definition 5 Let $M$ be an $(n+1)$-dimensional lightlike submanifold of a Lorentzian manifold. The submanifold $M$ is called co-screen locally conformal on a coordinate neighborhood $\mathcal{U}$ if there exists a non-zero smooth function $\varphi^{c}$ such that

$$
A_{N} X=\varphi^{c} A_{u_{\alpha}} X, \quad \forall X \in \Gamma(T M),
$$

for any null transversal vector field $N \in \Gamma(\operatorname{ltr}(T M))$ and $\alpha \in\{1, \ldots m\}$.

We now state the following theorem to characterize the co-screen conformal 1-lightlike submanifolds.

Theorem 6 Let $(M, g, S(T M))$ be a 1-lightlike submanifold of a Lorentzian manifold, then M is co-screen conformal if and only if

$$
C(X, P Y)=\varphi^{c} D^{\alpha}(X, P Y), \text { for all } X, Y \in \Gamma(T M),
$$

where $\varphi^{c}$ is a non-zero smooth function on $M$.

Proof. Let $(M, g, S(T M))$ be a 1-lightlike submanifold of a Lorentzian manifold. Then, it follows (17) and (18), we have

$$
C(X, P Y)=g\left(A_{N} X, P Y\right)=\varphi^{c} g\left(A_{u_{\alpha}} X, P Y\right)=\varphi^{c} D^{\alpha}(X, P Y),
$$

for any $X, Y \in \Gamma(T M)$.

Conversely, since the shape operator $A_{N}$ is $S(T M)$-valued, $\rho_{\alpha}=0$ for all $\alpha \in\{1, \ldots m\}$. Thus, we obtain $C(X, P Y)=$ $\varphi^{c} D^{\alpha}(X, P Y)$ which implies that $M$ is co-screen locally conformal.

Theorem 7 The conditions given in Theorem 4 are always satisfied for any co-screen locally conformal 1-lightlike submanifold of a Lorentzian manifold.

Example 8 Consider in $R_{1}^{7}$ with signature $(-,+,+,+,+,+,+)$ a submanifold $M$ given by the equations

$$
x_{4}=\left(x_{1}^{2}-x_{2}^{2}\right)^{\frac{1}{2}}, \quad x_{3}=\left(1-x_{5}^{2}\right)^{\frac{1}{2}}, \quad x_{6}=\left(1-x_{7}^{2}\right)^{\frac{1}{2}}, x_{2}, x_{5}, x_{7}>0 .
$$


Then, we have

$$
\begin{aligned}
T M= & \operatorname{Span}\left\{\xi=x_{1} \partial x_{1}+x_{2} \partial x_{2}+x_{4} \partial x_{4},\right. \\
& U_{1}=x_{4} \partial x_{1}+x_{1} \partial x_{4}, U_{2}=-x_{5} \partial x_{3}+x_{3} \partial x_{5}, \\
& \left.U_{3}=x_{6} \partial x_{7}+x_{7} \partial x_{6}\right\},
\end{aligned}
$$

and

$$
T M^{\perp}=\operatorname{Span}\left\{\xi, u_{1}=x_{3} \partial x_{3}+x_{5} \partial x_{5}, u_{2}=-x_{6} \partial x_{6}+x_{7} \partial x_{7}\right\} .
$$

Thus, $\operatorname{Rad} T M=\operatorname{Span}\{\xi\}$ is a distribution on $M$ and $S\left(T M^{\perp}\right)=\operatorname{Span}\left\{u_{1}, u_{2}\right\}$. Hence, $M$ is a 1-lightlike submanifold of $R_{1}^{7}$ with $S(T M)=\operatorname{Span}\left\{U_{1}, U_{2}, U_{3}\right\}$. Also, the lightlike transversal bundle $\operatorname{ltr}(T M)$ is spanned by

$$
N=\frac{1}{2 x_{1}}\left\{-x_{1} \partial x_{1}+x_{2} \partial x_{2}+x_{4} \partial x_{4}\right\}
$$

By direct calculations, we get the manifold $(M, g, S(T M))$ isn't co-screen conformal since $\varphi^{c}$ can't be vanishing function.

Example 9 Let $\widetilde{M}=R_{1}^{6}$ be be a semi-Euclidean space of signature $(-,+,+,+,+,+,+,+)$ with respect to the canonical basis

$$
\left\{\partial x_{1}, \partial x_{2}, \partial x_{3}, \partial x_{4}, \partial x_{5}, \partial x_{6}\right\}
$$

Let $M$ be a submanifold of $R_{1}^{8}$ given by

$$
\begin{aligned}
& x_{1}=\sinh u^{1}, \quad x_{2}=\cosh u^{1}, \quad x_{3}=u^{1}, x_{4}=u^{2}, \\
& x_{5}=\frac{1}{\sqrt{2}} u^{3}, \quad x_{6}=-\frac{1}{\sqrt{2}} u^{3},
\end{aligned}
$$

where all of $u^{1}, u^{2}, u^{3}, u^{4}$ are non-vanishing coordinate functions. Then, we have

$$
\begin{aligned}
& \operatorname{Rad} T M=\operatorname{Span}\left\{\xi=\cosh u^{1} \partial x_{1}+\sinh u^{1} \partial x_{2}+\partial x_{3}\right\} \\
& S(T M)=\operatorname{Span}\left\{e_{1}=\partial x_{4}, \quad e_{2}=\frac{1}{\sqrt{2}} \partial x_{5}-\frac{1}{\sqrt{2}} \partial x_{6}\right\} \\
& \operatorname{ltr}(T M)=\operatorname{Span}\left\{N=-\frac{1}{2} \cosh u^{1} \partial x_{1}-\frac{1}{2} \sinh u^{1} \partial x_{2}+\frac{1}{2} \partial x_{3}\right\} \\
& \operatorname{tr}(T M)=\operatorname{Span}\left\{u_{1}=\frac{1}{\sqrt{2}} \partial x_{5}+\frac{1}{\sqrt{2}} \partial x_{6}, \quad u_{2}=\sinh u^{1} \partial x_{1}+\cosh u^{1} \partial x_{2}\right\}
\end{aligned}
$$

By straightforward computations, it can be obtained that the submanifold is co-screen conformal with $\varphi^{c}$ is arbitrary.

Now, we give the following:

Theorem 10 Let $(M, g, S(T M))$ be a totally geodesic, totally umbilical or minimal screen locally conformal 1 -lightlike submanifold of a Lorentzian manifold $(\widetilde{M}, \widetilde{g})$. Any leaf $M^{\prime}$ of $S(T M)$ immersed in $\widetilde{M}$ as a $(m+2)$ dimensional non-degenerate submanifold if and only if the following assertions must be occurred:

a) $M$ is irrotational.

b) $M$ is co-screen locally conformal.

Proof. Let $M$ is a co-screen locally conformal irrotational 1-lightlike submanifold. Suppose that $X, Y$ be tangent vector fields of the leaf $M^{\prime}$ of a screen distribution and $h^{\prime}$ is its second fundamental form in $\widetilde{M}$. If we put (16), (17), (18) and (39) in (34) we get

$$
\widetilde{\nabla}_{X} Y=\nabla_{X}^{*} Y+g\left(A_{\xi}^{*} X, Y\right)(\varphi \xi+N)+\sum_{\alpha=1}^{n} \widetilde{g}\left(A_{u_{\alpha}} X, Y\right) u_{\alpha},
$$


which implies that

$$
h^{\prime}(X, Y)=B(X, Y)\left(\varphi \xi+N+u_{\alpha}\right), \text { for any } \alpha=\{1, \ldots, m\}
$$

and so

$$
h^{\prime}(X, Y)=\sqrt{\varphi} B(X, Y)\left(\sqrt{\varphi} \xi+\frac{1}{\sqrt{\varphi}} N+u_{\alpha}\right)
$$

where $\left(\sqrt{\varphi} \xi+\frac{1}{\sqrt{\varphi}} N+u_{\alpha}\right)$ is a unit spacelike vector field on $M^{\prime}$. Since $M$ is irrotational $D^{\alpha}(X, \xi)=0$ for all $\alpha \in\{1, \ldots, m\}$ and $B(X, \xi)=0$ for all $X \in \Gamma\left(T M^{\prime}\right)$. Therefore, the leaf $M^{\prime}$ of $S(T M)$ immersed in $\widetilde{M}$ as a $(m+2)$-dimensional non-degenerate submanifold. The proof of the converse part is straightforward.

Corollary 11 If $(M, g, S(T M))$ is a lightlike hypersurface of a Lorentzian manifold $(\widetilde{M}, \widetilde{g})$. Then, any leaf $M^{\prime}$ of $S(T M)$ immersed in $\widetilde{M}$ as a 2-dimensional non-degenerate submanifold.

Remark 12 The above corollary is also valid for lightlike hypersurfaces of a semi-Riemannian manifold that proved in (Atindogbe, Ezin E Tossa, 2006).

Theorem 13 Let $(M, g, S(T M))$ be a co-screen conformal 1-lightlike submanifold of a Lorentzian manifold. The co-screen distribution $S\left(T M^{\perp}\right)$ is a conformal Killing on $\widetilde{M}$ if and only if $S(T M)$ is totally umbilical.

Proof. Let $\widetilde{L}$ denote the Lie derivative on $\widetilde{M}$. If $S\left(T M^{\perp}\right)$ is conformal Killing on $\widetilde{M}$, then

$$
\left(\widetilde{L}_{u_{\alpha}} \widetilde{g}\right)(X, Y)=\widetilde{g}\left(\widetilde{\nabla}_{X} u_{\alpha}, Y\right)+\widetilde{g}\left(X, \widetilde{\nabla}_{Y} u_{\alpha}\right)=\delta g(X, Y),
$$

for any $u_{\alpha} \in \Gamma\left(S\left(T M^{\perp}\right)\right)$ and $X, Y \in \Gamma(T M)$. Here, $\delta$ is a smooth function. Putting (17) in (48), we get

$$
\left(\widetilde{L}_{u_{\alpha}} \widetilde{g}\right)(X, Y)=-2 D^{\alpha}(X, Y)=\delta g(X, Y),
$$

for any $X, Y \in \Gamma(T M)$. Since $M$ is co-screen conformal, we obtain

$$
C(X, Y)=-\frac{\delta}{2 \varphi} g(X, Y),
$$

which shows that $S(T M)$ is totally umbilical.

Now, we assume that $S(T M)$ is totally umbilical. Then,

$$
C(X, Y)=\lambda^{\prime} g(X, Y), \quad \forall X, Y \in \Gamma(S(T M))
$$

where $\lambda^{\prime}$ is a smooth function. Putting $\lambda^{\prime}=-\frac{\delta}{2 \varphi}$, it is clear that $S\left(T M^{\perp}\right)$ is a conformal Killing on $\widetilde{M}$.

Corollary 14 Let $(M, g, S(T M))$ be co-screen conformal 1-lightlike submanifold of a Lorentzian manifold. The co-screen distribution $S\left(T M^{\perp}\right)$ is a Killing distribution on $\widetilde{M}$ if and only if $S(T M)$ is totally geodesic and $M$ is minimal.

Using similar proof method of Theorem 3.10 in (Wang \& Liu, 2013) we have also the following:

Theorem 15 Let $(M, g, S(T M))$ be a co-screen conformal 1-lightlike submanifold of a Lorentzian manifold. Then,

1) Any leaf of $(S T M)$ is totally geodesic on $M$.

2) The submanifold $M$ is a lightlike product manifold of $M^{\prime}$ and $F$ where $M^{\prime}$ is a leaf of $S(T M)$ and $F$ is a null curve of $M$.

3) $D^{\alpha}=0$ on $S(T M)$ for all $\alpha \in\{1, \ldots, m\}$.

\section{Ricci Curvature on Co-screen Conformal 1-lightlike Submanifolds}

In this section, we study on the sectional curvature, the null sectional curvature and the induced Ricci curvature on co-screen conformal 1-lightlike submanifolds of a Lorentzian manifold. We begin this section herewith the following lemma.

Lemma 16 Let $(M, g, S(T M))$ be a co-screen conformal 1-lightlike submanifold of a Lorentzian manifold. Let us denote the Riemannian curvature tensors $R$ and $\widetilde{R}$ of the submanifold $M$ and the ambient manifold $\widetilde{M}$, respectively. 
Then, we have the following relations:

$$
\begin{aligned}
\widetilde{g}(\widetilde{R}(X, Y) P Z, P W)= & g(R(X, Y) P Z, P W)+B(X, P Z) C(Y, P W)-B(Y, P Z) C(X, P W) \\
& +\frac{1}{\left(\varphi^{c}\right)^{2}}[C(X, P Z) C(Y, P W)-C(Y, P Z) C(X, P W)], \\
\widetilde{g}(\widetilde{R}(X, Y) Z, N)= & \widetilde{g}(R(X, Y) Z, N)+\frac{1}{\varphi^{c}}\left[C(X, Z) \rho_{\alpha}(Y)-C(Y, Z) \rho_{\alpha}(X)\right] \\
& +B(X, Z) \eta\left(A_{N} Y\right)-B(Y, Z) \eta\left(A_{N} X\right), \\
\widetilde{g}(\widetilde{R}(X, Y) \xi, N)= & \widetilde{g}(R(X, Y) \xi, N)+\sum_{\alpha=1}^{m} \rho_{\alpha}(X) \varepsilon_{\alpha}(Y)-\rho_{\alpha}(Y) \varepsilon_{\alpha}(X), \\
\widetilde{g}(\widetilde{R}(X, Y) Z, \xi)= & \left(\nabla_{X} B\right)(Y, Z)-\left(\nabla_{Y} B\right)(X, Z)+B(Y, Z) \rho(X)-B(X, Z) \rho(Y) \\
& +\frac{1}{\varphi^{c}}\left[\sum_{\alpha=1}^{m} C(Y, Z) \varepsilon_{\alpha}(X)-C(X, Z) \varepsilon_{\alpha}(Y)\right],
\end{aligned}
$$

where $X, Y, Z, W \in \Gamma(T M), N \in \Gamma(\operatorname{ltr}(T M))$ and $\xi \in \operatorname{Rad} T M$.

Let $(M, g, S(T M))$ be $(n+1)$-dimensional co-screen conformal 1-lightlike submanifold, $\left\{e_{1}, \ldots, e_{n}, \xi, N, u_{1}, \ldots, u_{m}\right\}$ be a quasi-orthonormal basis on $(\widetilde{M}, \widetilde{g})$. From (52) and (53), we have

$$
\begin{aligned}
\widetilde{g}\left(\widetilde{R}\left(e_{i}, X\right) Y, e_{i}\right)= & g\left(R\left(e_{i}, X\right) Y, e_{i}\right)+B\left(e_{i}, Y\right) C\left(X, e_{i}\right)-B(X, Y) C\left(e_{i}, e_{i}\right) \\
& +\frac{1}{\left(\varphi^{c}\right)^{2}}\left[C\left(e_{i}, Y\right) C\left(X, e_{i}\right)-C(X, Y) C\left(e_{i}, e_{i}\right)\right]
\end{aligned}
$$

and

$$
\widetilde{g}\left(\widetilde{R}\left(e_{i}, \xi\right) Y, N\right)=\widetilde{g}(R(X, \xi) Y, N)
$$

Theorem 17 Let $(M, g, S(T M))$ be an $(n+1)$-dimensional co-screen conformal 1-lightlike submanifold of a Loretzian manifold $(\widetilde{M}, \widetilde{g})$. Then, the induced Ricci curvature tensor of $M$ is symmetric.

Proof. From (20), (56) and (57) we get

$$
\begin{aligned}
\operatorname{Ric}(X, Y)= & \sum_{i=1}^{n} g\left(R\left(e_{i}, X\right) Y, e_{i}\right)+\widetilde{g}(R(X, \xi) Y, N) \\
= & \widetilde{\operatorname{Ric}}_{S(T M)}(X, Y)+\widetilde{g}(\widetilde{R}(X, \xi) Y, N)+\mu^{*}\left[B(X, Y)+\frac{1}{\left(\varphi^{c}\right)^{2}} C(X, Y)\right] \\
& -\sum_{i=1}^{n}\left[B\left(Y, e_{i}\right) C\left(X, e_{i}\right)+\frac{1}{\left(\varphi^{c}\right)^{2}} C\left(Y, e_{i}\right) C\left(X, e_{i}\right)\right],
\end{aligned}
$$

where $\widetilde{\operatorname{Ric}}_{S(T M)}(X, Y)$ is $n$-plane section Ricci curvature of $T_{p} \widetilde{M}$ for any $X, Y \in \Gamma(T M)$. Since the operator $C$ is symmetric on these submanifolds, it is clear that the Ricci tensor is symmetric.

Using (56) we have the following theorem:

Theorem 18 If $(M, g, S(T M))$ is co-screen conformal 1-lightlike submanifold of a Lorentzian space form $\widetilde{M}(c)$ with constant curvature $c$. Then, the null sectional curvature of $M$ is given by

$$
K_{\xi}(\Pi)=D^{\alpha}(\xi, \xi) D^{\alpha}\left(e_{i}, e_{i}\right)-\left[D^{\alpha}\left(\xi, e_{i}\right)\right]^{2}, \text { for any } \alpha \in\{1, \ldots, m\},
$$

where $\Pi=\operatorname{Span}\left\{\xi, e_{i}\right\}$ is a degenerate plane section of $T_{p} M$.

We note that if the submanifold $(M, g, S(T M))$ is irrotational then the null sectional curvature given by (59) vanishes identically.

Definition 19 We define the screen Ricci curvature at a unit vector $e_{i} \in \Gamma(S(T M))$ as

$$
\operatorname{Ric}_{S(T M)}\left(e_{i}\right)=\sum_{j=1}^{n} K_{i j}, \quad 1 \leq i \neq j \leq n,
$$


Since $K_{i j}$ are symmetric for all $i, j \in\{1, \ldots, n\}$ in co-screen conformal 1-lightlike submanifolds of a Lorentzian manifold, it is clear that the screen Ricci curvature is well defined.

We note that the screen Ricci curvature vanishes identically if $n=1$, it is equal to the sectional curvature if $n=2$.

Theorem 20 Let $(M, g, S(T M))$ be a 4-dimensional co-screen conformal 1-lightlike submanifold of a Lorentzian manifold. The screen Ricci curvature $\operatorname{Ric}_{S(T M)}$ is constant at every unit vector on $\Gamma(S(T M))$ if and only if the following conditions are occurred.

a) The sectional curvature function on $M$ is constant.

b) Any leaf $M^{\prime}$ of $S(T M)$ immersed in $\widetilde{M}$ is $(m+2)$-dimensional non-degenerate submanifold with constant curvature.

Proof. Let $\left\{e_{1}, e_{2}, e_{3}\right\}$ be an orthonormal basis of $\Gamma(S(T M))$. If $\operatorname{Ric}_{S(T M)}$ is constant, then

$$
\begin{aligned}
& \operatorname{Ric}_{S(T M)}\left(e_{1}\right)=K_{12}+K_{13}=\lambda, \\
& \operatorname{Ric}_{S(T M)}\left(e_{2}\right)=K_{21}+K_{23}=\lambda, \\
& \operatorname{Ric}_{S(T M)}\left(e_{3}\right)=K_{31}+K_{32}=\lambda,
\end{aligned}
$$

where $\lambda$ is a constant. Thus, we have

$$
K_{12}=\frac{1}{2}\left[\operatorname{Ric}_{S(T M)}\left(e_{1}\right)+\operatorname{Ric}_{S(T M)}\left(e_{2}\right)-\operatorname{Ric}_{S(T M)}\left(e_{3}\right)\right]=\frac{1}{2} \lambda,
$$

which shows that the sectional curvature is constant and any leaf $M^{\prime}$ of $S(T M)$ immersed in $\widetilde{M}$ is $(m+2)$ dimensional non-degenerate submanifold with constant curvature. The proof of the converse part is straightforward.

Theorem 21 Let $(M, g, S(T M))$ be an $(n+1)$-dimensional co-screen conformal 1-lightlike submanifold of a semiEuclidean space with the screen Ricci curvature on the submanifold vanishes identically. Then, at least one of the following situations are occurred:

a) $\mu_{1} C(X, X)=\sum_{i=1}^{n} B\left(e_{i}, X\right) C\left(e_{i}, X\right)$ for all $X \in \Gamma(S(T M))$.

b) $\varphi^{c}=\mp 1$.

Proof. If we take trace in (56), we have

$$
\left(\frac{\left(\varphi^{c}\right)^{2}-1}{\left(\varphi^{c}\right)^{2}}\right) \sum_{i=1}^{n}\left[B\left(e_{i}, X\right) C\left(e_{i}, X\right)\right]-\mu_{1} C(X, X)=0,
$$

which implies that

$$
\mu_{1} C(X, X)=\sum_{i=1}^{n} B\left(e_{i}, X\right) C\left(e_{i}, X\right)
$$

or

$$
\varphi^{c}=\mp 1
$$

This is proof of the theorem.

Theorem 22 Let $(M, g, S(T M))$ be an $(n+1)$-dimensional flat co-screen conformal 1-lightlike submanifold with $B=0$ of a semi-Euclidean space. Then, the local shape operator of $M$ takes the form as follows:

$$
A_{\xi}^{*}=\left(\begin{array}{cccc}
a & -a & \cdots & -a \\
-a & a & \cdots & -a \\
\vdots & \vdots & \ddots & \vdots \\
-a & -a & \cdots & a
\end{array}\right),
$$

for a is a real number. 
Proof. Since $M$ is flat, using (53) and (54) one can write

$$
\begin{gathered}
D(Y, Z) \varepsilon_{\alpha}(X)=D(X, Z) \varepsilon_{\alpha}(Y), \\
D(X, Z) \rho_{\alpha}(Y)=D(Y, Z) \varepsilon_{\alpha}(X),
\end{gathered}
$$

i.e

$$
D(Y, Z)\left[\varepsilon_{\alpha}(X)-\rho_{\alpha}(Y)\right]=D(X, Z)\left[\varepsilon_{\alpha}(Y)-\rho_{\alpha}(X)\right] .
$$

for all $X, Y, Z \in T M$. If we put $Y=X+Z$ then we have

$$
D(Z, Z)\left[\varepsilon_{\alpha}(X)-\rho_{\alpha}(X)\right]=D(X, Z)\left[\varepsilon_{\alpha}(Z)-\rho_{\alpha}(Z)\right],
$$

i.e

$$
\frac{\varepsilon_{\alpha}(X)-\rho_{\alpha}(X)}{\varepsilon_{\alpha}(Z)-\rho_{\alpha}(Z)}=\frac{D(X, Z)}{D(Z, Z)} .
$$

By using similar method, one has

$$
\frac{\varepsilon_{\alpha}(X)-\rho_{\alpha}(X)}{\varepsilon_{\alpha}(Y)-\rho_{\alpha}(Y)}=\frac{D(X, Z)}{D(Z, Z)},
$$

which implies that $D(Y, Y)=D(Z, Z)$. Taking into consideration (52) it can be obtained $D(Y, Y)=-D(X, Y)$. Thus the shape operator $A_{\xi}^{*}$ takes the form as (62).

Definition 23 Let $(M, g, S(T M))$ be an $(n+1)$-dimensional co-screen conformal 1-lightlike submanifold of a Loretzian manifold $(\widetilde{M}, \widetilde{g})$. Let $\left\{e_{1}, \ldots, e_{n}\right\}$ be an orthonormal basis of $\Gamma(S(T M))$. We define the screen scalar curvature at a point $p \in M$ as

$$
r_{S(T M)}(p)=\frac{1}{2} \sum_{i, j=1}^{n} K_{i j}
$$

Using (52) and (67), we state the following lemma:

Lemma 24 Let $(M, g, S(T M))$ be an $(n+1)$-dimensional co-screen conformal 1-lightlike submanifold of a Loretzian manifold $(\widetilde{M}, \widetilde{g})$. Let $\left\{e_{1}, \ldots, e_{n}\right\}$ be an orthonormal basis of $\Gamma(S(T M))$. Then we have

$$
2 r_{S(T M)}(p)=2 \widetilde{r}_{S(T M)}(p)+\mu^{*}\left(\mu^{*}+\mu_{1}\right)-\sum_{i, j=1}^{n}\left[B_{i j} C_{i j}+\left(\frac{C_{i j}}{\varphi^{c}}\right)^{2}\right],
$$

where $B_{i j}=B\left(e_{i}, e_{j}\right)$ and $C_{i j}=C\left(e_{i}, e_{j}\right)$ for all $i, j \in\{1, \ldots, n\}$.

Let us denote

$$
|B|^{2}=\sum_{i, j}^{n}\left(B_{i j}\right)^{2} \text { and }|C|^{2}=\sum_{i, j}^{n}\left(C_{i j}\right)^{2} .
$$

We note that both the second fundamental forms $B$ and $C$ are independent of the screen distribution in co-screen conformal 1-lightlike submanifolds of a Lorentzian manifold it is clear that the norms of these operators are welldefined.

Theorem 25 Let $(M, g, S(T M))$ be an $(n+1)$-dimensional co-screen conformal 1-lightlike submanifold of a Loretzian manifold $(\widetilde{M}, \widetilde{g})$. Then, we have

$$
2 r_{S(T M)}(p) \leq 2 \widetilde{r}_{S(T M)}(p)+\mu^{*}\left(\mu^{*}+\mu_{1}\right)+\frac{1}{2}|B|^{2}+\frac{1-2\left(\varphi^{c}\right)^{2}}{\left(\varphi^{c}\right)^{2}}|C|^{2} .
$$

The equality case of (70) holds for the point $p \in M$ if and only if either the submanifold $M$ is totally geodesic or is also screen locally conformal with $\varphi=-1$. 
Proof. If we put

$$
\sum_{i, j=1}^{n} B_{i j} C_{i j}=\frac{1}{2}\left\{\sum_{i, j=1}^{n}\left(B_{i j}+C_{i j}\right)^{2}-\left(B_{i j}\right)^{2}-\left(C_{i j}\right)^{2}\right\}
$$

in Lemma 24, we get

$$
\begin{aligned}
2 r_{S(T M)}(p)= & 2 \widetilde{r}_{S(T M)}(p)+\mu^{*}\left(\mu^{*}+\mu_{1}\right)-\frac{1}{2} \sum_{i, j=1}^{n}\left(B_{i j}+C_{i j}\right)^{2} \\
& +\frac{1}{2} \sum_{i, j=1}^{n}\left(B_{i j}\right)^{2}+\frac{1}{2\left(\varphi^{c}\right)^{2}} \sum_{i, j=1}^{n}\left(C_{i j}\right)^{2}
\end{aligned}
$$

which implies (70) inequality. The equality case of (70) holds for the point $p \in M$ if and only if

$$
\sum_{i, j=1}^{n}\left(B_{i j}+C_{i j}\right)^{2}=0,
$$

which shows that the submanifold $M$ is totally geodesic or is also screen locally conformal with $\varphi=-1$.

Taking into consideration (70), we have also the following theorem:

Theorem 26 Let $(M, g, S(T M))$ be an $(n+1)$-dimensional co-screen conformal 1-lightlike submanifold of a Loretzian manifold $(\widetilde{M}, \widetilde{g})$. Then, we have

$$
2 r_{S(T M)}(p) \leq 2 \widetilde{r}_{S(T M)}(p)+\mu^{*}\left(\mu^{*}+\mu_{1}\right)-\frac{1}{2}(\operatorname{trace} \bar{A})^{2}+\frac{1}{2}|B|^{2}+\frac{1-2\left(\varphi^{c}\right)^{2}}{\left(\varphi^{c}\right)^{2}}|C|^{2},
$$

where

$$
\bar{A}=\left(\begin{array}{cccc}
B_{11}+C_{11} & B_{12}+C_{12} & \cdots & B_{1 n}+C_{1 n} \\
B_{21}+C_{21} & B_{22}+C_{22} & \cdots & B_{2 n}+C_{2 n} \\
\vdots & & & \\
B_{n 1}+C_{n 1} & B_{n 2}+C_{n 2} & \cdots & B_{n n}+C_{n n}
\end{array}\right) .
$$

The equality case of (74) holds for the point $p \in M$ if and only if $B(X, Y)=-C(X, Y)$ for all mutually orthogonal vectors $X, Y \in \Gamma(S(T M))$.

\section{Acknowledgements}

This work is supported by 113F388 coded project in the Scientific and Technological Research Council of Turkey (TÜBİTAK). The authors are thankful to the referee for valuable suggestions for improvement of the paper.

\section{References}

Akivis, M. A., \& Goldberg, V. V. (1998). The geometry of lightlike hypersurfaces of the de Sitter space. Acta Appl. Math., 53, 297-328. http://dx.doi.org/10.1023/A:1006062604176

Akivis, M. A., \& Goldberg, V. V. (1999). Lightlike hypersurfaces on manifolds endowed with a conformal structure of Lorentzian signature. Acta Appl. Math., 57, 255-285. http://dx.doi.org/10.1023/A:1006244706787

Akivis, M. A., \& Goldberg, V. V. (2000). On some methods of construction of in-variant normalizations of lightlike hypersurfaces. Differential Geometry and its Applications, 12(2), 121-143. http://dx.doi.org/10.1016/S09262245(00)00008-5

Albujer A. L., \& Haesen, S. (2010). A geometrical interpretation of the null sectional curvature. J. of Geo. and Phys., 60(3), 471-476. http://dx.doi.org/10.1016/j.geomphys.2009.11.002

Atindogbe, C., \& Duggal K. L. (2004). Conformal screen on lightlike hypersurfaces. Int. J. Pure Appl. Math., 11(4), 421-442.

Atindogbe, C., Ezin, J.-P., \& Tossa J. (2006). Lightlike Einstein hypersurfaces in Lorentzian manifolds with constant curvature. Kodai Math. J., 29(1), 58-71. 
Beem, J. K., Ehrlich, P. E., \& Easley, K. L. (1996). Global Lorentzian geometry. Marcel Dekker Inc., New York, Second Edition.

Bejan, C. L., \& Duggal, K. L. (2005). Global lightlike manifolds and harmonicity. Kodai Math. J., 28, 131-145. http://dx.doi.org/10.2996/kmj/1111588042

Bolós, V. J. (2005). Geometric description of lightlike foliations by an observer in general relativity. Math. Proc. Camb. Phil. Soc., 139, 181-192. http://dx.doi.org/10.1017/S0305004105008571

Bonnor, W. B. (1972). Null hypersurfaces in Minkowski spacetime. Tensor N. S., 24, 329-345.

Duggal, K. L., \& Bejancu, A. (1996). Lightlike submanifolds of semi-Riemannian manifolds and applications. Mathematics and Its Applications, Kluwer Academic Publisher, Dordrecht, 364, 1996.

Duggal, K. L., \& Sahin, B. (2004). Screen conformal half-lightlike submanifolds. Int. J. Math. and Math. Sci., 68, 3737-3753. http://dx.doi.org/10.1155/S0161171204403342

Duggal K. L., (2007). On canonical screen for lightlike submanifolds of codimensional two, Cent. Eur. J. Math., 5(4), 710-719.

Duggal, K. L., \& Jin, D. H. (2007). Null curves and hypersurfaces of semi-Riemannian manifolds. World Scientific. http://dx.doi.org/10.1142/6449

Duggal, K. L., \& Sahin, B. (2006). Generalized Couchy Riemann lightlike submanifolds. Acta Math. Hungar, 112(1-2), 113-136.

Duggal K. L., \& Sahin B. (2010). Differential Geometry of Lightlike Submanifolds. Birkhäuser Verlag AG, Germany.

Gülbahar, M., Kılıç E., \& Keleş S. (2013a). Chen-like inequalities on lightlike hypersurfaces of a Lorentzian manifold. J. of Ineq. and Appl., 2013:266.

Gülbahar, M., Kılıç, E., \& Keleş, S. (2013b). Some inequalities on screen homothetic lightlike hypersurfaces of a Lorentzian manifold. Taiwan. J. Math., 17, 2083-2100.

Jin, D. H. (2009a). Screen conformal lightlike hypersurfaces of a semi-Riemannian space form. J. Korea Soc. Math. Educ. Ser. B: Pure Appl. Math., 16(3), 271-276.

Jin, D. H. (2009b). Screen conformal lightlike real hypersurfaces of an indefinite complex space form. Bull. Korean Math. Soc., 341-353.

Jin, D. H. (2009c). A characterization of screen conformal half lightlike submanifolds. Honam Mathematical J., $31(1), 17-23$.

Jin, D. H. (2010). Screen conformal Einstein lightlike hypersurfaces of a Lorentzian space form. Commun. Korean Math. Soc., 25(2), 225-234. http://dx.doi.org/10.4134/CKMS.2010.25.2.225

Jin, D. H. (2010b). Geometry of screen conformal real half lightlike submanifolds. Bull. Korean Math. Soc., 47(4), 701-714

Jin, D. H. (2011). Half lightlike submanifolds of an indefinite Sasakian manifold, J. of the Korea Soc. of Math. Edu. B, 18(2), 173-183.

Jin, D. H. (2014). A characterization theorem for lightlike hypersurfaces of semi-Riemannian manifolds of quasiconstant curvatures. East Asian Math. J., 30(1), 015-022.

Kupeli, D. N. (1996). Singular Semi-Riemannian Geometry, Kluwer Academic, 366.

Leistner, T. (2006). Screen bundles of Lorentzian manifolds and some generalizations of pp-waves, J. Geom. Phys., 56(10), 2117-2134. http://dx.doi.org/10.1016/j.geomphys.2005.11.010

Massamba, F. (2008). On weakly Ricci symmetric lightlike hypersurfaces of indefinite Sasakian manifolds. SUT J. Math., 44(2), 181-201.

Massamba, F. (2012). Screen almost conformal lightlike geometry in indefinite Kenmotsu space forms. Int. Elec. J. of Geom., 5(2), 36-58.

Sahin, B. (2005). Warped Product Lightlike Submanifolds, Sarajevo J. of Math., 1(14), 251-260. 
Sahin, B. (2007). Screen conformal submersions between lightlike manifolds and semi-Riemannian manifolds and their harmonicity. Int. J. Geom. Methods Mod. Phys., 4(6), 987-1003. http://dx.doi.org/10.1142/S0219887807002405

Wang, W., Wang, Y., \& Liu, X. (2013). Screen transversal conformal half-lightlike submanifolds. Ann. of the Univ. of Craiova, Mathematics and Computer Science Series, 40(2), 140-147.

Wang, Y., \& Liu, X. (2013). Co-screen conformal half lightlike submanifolds. Tamkang J. Math., 44(4), 431-444. http://dx.doi.org/10.5556/j.tkjm.44.2013.1232

\section{Copyrights}

Copyright for this article is retained by the author(s), with first publication rights granted to the journal.

This is an open-access article distributed under the terms and conditions of the Creative Commons Attribution license (http://creativecommons.org/licenses/by/3.0/). 